\title{
Obtención de Biopelículas Binarias Activas y su Efecto en la Vida Útil Microbiológica del Camarón Blanco (Penaeus vannamei)
}

\author{
Rafael E. González Cuello*, Jasbeidy Castro Bravo y Yohana Caro Pérez \\ Universidad de Cartagena, Piedra de Bolívar - Av Del Consulado, Calle 30 № 48-157., Colombia. \\ (e-mail: rgonzalezc1@unicartagena.edu.co)
}

* Autor a quien debe ser dirigida la correspondencia

Recibido Ene. 5, 2015; Aceptado Mar. 12, 2015; Versión final Abr. 25, 2015, Publicado Oct. 2015

\begin{abstract}
Resumen
En la presente investigación se elaboraron biopelículas activas a base de alginato, almidón de maíz y extracto acuoso de toronjil (Melissa officinalis $L$ ), evaluando su incidencia en la vida útil microbiológica del camarón blanco del pacifico (Penaeus vannamei). Para la estimación de la vida útil se realizaron cinéticas de crecimiento de microorganismos indicadores (aerobios mesófilos) modeladas matemáticamente utilizando la ecuación de Baranyi y Robert utilizando el programa DMFIT para obtener los parámetros cinéticos de crecimiento microbiano a diferentes temperaturas. Finalmente la ecuación de MonodHinshelwood fue utilizada para estimar la vida útil de los camarones. Los resultados indicaron que es posible utilizar biopelículas a base de alginato, almidón de maíz y como sustancia antimicrobiana extracto acuoso de toronjil para la conservación de camarones (Penaeus vannamei), debido a que se obtuvieron incrementos del $33 \%$ aproximadamente en la vida útil de camarones (Penaeus vannamei) recubiertos con las biopelículas.
\end{abstract}

Palabras clave: alginato, almidón de maíz, biopelículas activas, vida útil

\section{Obtaining Active Binary Biofilm and its Effect on Microbiological Shelf Life of White Shrimp (Penaeus Vannamei)}

\begin{abstract}
In this research active biofilms based on alginate, cornstarch and water extract of lemon balm (Melissa officinalis $L$ ) were developed, assessing their impact on the microbiological shelf life of Pacific white shrimp (Penaeus vannamei). Microbial growth kinetics using as indicators microorganism aerobic mesophilic bacterial modeled mathematically through Baranyi y Robert equation by the program DMFIT were used for determinate the microbial growth parameters at different temperatures. Finally Hinshelwood - Monod equation was used to estimate the shelf life of the shrimp. The results indicated that is possible obtain binary biofilms containing aqueous extract of lemon balm for the shrimp conservation, because increments of 6 days were obtained in the shelf life of shrimp coated with biofilms
\end{abstract}

Keywords: alginate, cornstarch, active biofilm, shelf life 


\section{INTRODUCCIÓN}

El camarón es uno de los productos acuáticos mayoritariamente comercializados y consumidos a nivel mundial (Chen et al., 2013). El camarón es propenso al deterioro debido a su alto contenido de aminoácidos libres y otras sustancias solubles que pueden servir como nutrientes fácilmente digerible para el crecimiento microbiano (Zeng et al., 2005). Estudios sobre la vida útil de los productos marinos se han enfocado en la cuantificación de microorganismos, trimetilamina, compuestos volátiles, nitrógeno y oxidación lipídica (Ocano et al., 2009; Castillo et al, 2007) pero poco se ha enfocado en estudios de microbiología predictiva. Para la preservación de camarones el método más utilizado ha sido la refrigeración, aunque a bajas temperaturas el producto puede sufrir reacciones autolíticas propias del crecimiento de bacterias deteriorantes como Pseudomonas sp y Shewanella putrefaciens, contribuyendo así a la disminución de la calidad y tiempo de vida útil del camarón (Bono y Badalucco, 2012).

En la actualidad, existe una demanda por parte de los consumidores en cuanto a la elaboración de productos frescos libres de cualquier agente químico y con un elevado valor nutricional. Es debido a ello, que el uso de biopelículas activas surge como alternativa para mantener los productos alimenticios frescos por mayor tiempo disminuyendo el uso de materiales termoplásticos, los cuales presentan problemas ambientales. Las biopelículas activas crean una barrera a los gases reduciendo la permeabilidad e intercambio de moléculas con el ambiente, evitando el deterioro de los alimentos (Kuorwel et al., 2011). Los materiales utilizados para el desarrollo de biopelículas incluyen las proteínas, lípidos y polisacáridos (Embuscado y Huber, 2009). Dentro de los polisacáridos más utilizados se encuentran los alginatos, que son ampliamente utilizados en la industria alimentaria debido a sus propiedades gelificantes, viscosantes y atoxicas. Químicamente los alginatos son copolimeros lineales aniónicos compuestos de $\beta-D$ ácido manuronico $(M)$, y $\alpha$ - $L$ ácido guluronico $(G)$ unidos por enlace $\beta$ 1-4 y es estructurado por bloques que pueden ser homopolimericos ( $M \circ G$ ) o heteropolimericos (MG) (Lee y Mooney, 2012). Una de las aplicaciones más importantes de los alginatos en biotecnología, es la habilidad que tienen para formar geles, lo cual ha sido utilizado también para llevar a cabo procesos de microencapsulación (González et al., 2014). El almidón es el segundo polisacárido de mayor abundancia en la naturaleza después de la celulosa. Está formado por dos clases de moléculas: la amilosa, de estructura lineal, que se encuentra generalmente en un porcentaje de entre 20 y $30 \%$; y la amilopectina, de estructura ramificada, cuyo porcentaje varía entre el 70 y $80 \%$. Dentro de las aplicaciones del almidón en la industria alimenticia se encuentran sus propiedades gelificante, glaseante, humectante, estabilizante, texturizante y espesante. Para el desarrollo de biopelículas, el almidón ha recibido una considerable atención debido a su naturaleza biodegradable, buena capacidad formadora de películas y bajo costo (Jiménez et al., 2014; Liu, 2005). Además, múltiples sustancias se pueden incorporar durante la obtención de biopelículas con el fin de modificar algunas características de las biopelículas. De especial interés resulta la incorporación de agentes antimicrobianos para originar biopelículas activas con funciones determinadas (Chana-Thaworn et al., 2011; Shen y et al., 2010).

Por tal razón, como parte innovadora de las biopelículas se encuentra la incorporación de nuevos componentes entre los cuales se destacan los extractos acuosos, que actúan como compuestos antimicrobianos (Kuorwel et al., 2011). El toronjil (Melissa officinalis L.) pertenece a la familia Lamiaceae, es una planta medicinal muy estudiada, para la que se describen como principales compuestos los ácidos hydroxycinámicos y los aceites esenciales, donde los mayores constituyentes son los terpenoides como el citral, citronelal, geraniol, nerol y linalol (Esquivel y Vargas, 2007). Por lo mencionando anteriormente, el objetivo de la presente investigación es elaborar biopelículas activas utilizando polímeros funcionales adicionados con extractos acuosos de toronjil evaluando su efecto en la vida útil microbiológica del camarón blanco (Penaeus vannamei).

\section{MATERIALES Y METODOS}

El alginato de sodio fue suministrado por Modernist Pantry (EE.UU), El almidón de maíz, el glicerol y el toronjil se obtuvieron en el comercio local de la ciudad. Finalmente, las muestras de camarón blanco fueron proporcionadas por la pescadería Pez Caribe S.A. de la ciudad de Cartagena de indias-Colombia.

\section{Obtención del extracto acuoso}

Las plantas de Toronjil fueron lavadas con agua destilada e hiploclorito de sodio a 200 ppm durante 5 min y enjuagadas con abundante agua destilada. Las hojas fueron secadas al aire a temperatura ambiente y finalmente el material es triturado en una proporción 1:3 (agua destilada: hojas). La solución resultante es filtrada separando la porción acuosa de la vegetal en dos etapas, en la primera etapa se utilizó algodón como medio filtrante y en la segunda etapa se utilizaron filtros de hemicelulosa siguiendo la metodología propuesta por Herrera et al., (2006). El extracto de Toronjil (Melissa officinalis L.) fue almacenado en refrigeración en frascos color ámbar hasta su posterior uso. 


\section{Elaboración de las biopelículas}

El alginato y el almidón de maíz en proporciones (1:1) fueron disueltos en $39 \mathrm{~mL}$ de agua destilada, con ayuda de una plancha de calentamiento bajo agitación constante por 25- 30 minutos, una vez disuelta la solución se incorporaron $10 \mathrm{~mL}$ de glicerol como agente plastificante. La solución resultante fue sometida a calentamiento $90{ }^{\circ} \mathrm{C} / 10$ minutos en baño de maría, subsiguientemente la temperatura se disminuyó hasta alcanzar $30^{\circ} \mathrm{C}$ con el fin de adicionar el extracto acuoso de toronjil. Para la formación de las biopelículas fueron dispuestos volúmenes de $15 \mathrm{~mL}$ por placa, en cajas de petri previamente esterilizadas. El secado se realizó en una incubadora a $30^{\circ} \mathrm{C}$ por 24 horas, asegurando la dispersión y uniformidad de las biopelículas previo al secado (Rodríguez y Schobitz, 2009).

\section{Estimación de la vida útil microbiológica del camarón blanco}

Los camarones blanco utilizados en esta investigación fueron divididos en dos grupos. El primer grupo fue recubierto con las biopelículas activas, mientras que el segundo grupo fue tomado como muestras control (sin adición de biopelículas). Varias cinéticas de crecimiento microbiano fueron llevadas a cabo para los dos grupos de camarón blanco, utilizando como microorganismos indicadores el grupo aerobios mesófilos, los cuales están directamente correlacionados con la vida útil de la mayoría de sistemas alimentarios. Las cinéticas se realizaron a tres temperaturas $\left(17,27\right.$ y $\left.37^{\circ} \mathrm{C}\right)$ para cada grupo de camarones, tomando 15 puntos aproximadamente por cinética. Los datos obtenidos fueron modelados matemáticamente utilizando la ecuación de Baranyi y Roberts, (1994) (Ver Ecuación 1) por medio del programa de computo DMFit 2.0

$y(t)=y 0+\mu \max +\frac{1}{\mu \max } \operatorname{In}\left(\mathrm{e}^{-\mathrm{vt}}+\mathrm{e}^{-\mathrm{h} 0}-\mathrm{e}^{-\mathrm{vt}-\mathrm{ho}}\right)-\frac{1}{m} \operatorname{In}\left(1+\frac{e^{\operatorname{m\mu max}+\frac{1}{\mu \max } I n\left(e^{-v t}+e^{-h 0}-e^{-v t-h o}\right)}}{e^{m(y \max -y o)}}\right)$

Donde $y(t)$ es la concentración celular o el diámetro de las colonias, yo es la concentración o diámetro inicial, $\mu$ máx es la velocidad específica de crecimiento. $\left(\frac{1}{h}\right), m$ es un parámetro de curvatura para caracterizar la transición de la fase exponencial, $v$ es un parámetro de curvatura para caracterizar la transición a la fase exponencial y $h_{0}$ es un parámetro adimensional que cuantifica el estado fisiológico inicial de las células. Una vez obtenidos los parámetros cinéticos de crecimiento microbiano, se procedió a estimar la vida útil microbiológica utilizando la ecuación de Monod-Hinshelwood (ecuación 2).

ts $=\frac{\log N_{s}-\log N_{0}}{\log 2} x T_{d}$

Donde ts es el tiempo necesario para que se desarrolle la alteración en el alimento, Ns (ufc/g) es el valor correspondiente a la población de seguridad (valor máximo permisible antes de considerarse alterado el producto), $\mathrm{N}_{0}$ (ufc/g) es el valor correspondiente a la población inicial presente en el producto y Td es el tiempo de duplicación de la población alterante especifica.

\section{Análisis Microbiológicos}

Se tomaron $11 \mathrm{~g}$ de las muestras (Penaeus vannamei) y se adicionaron en $99 \mathrm{~mL}$ de agua de peptona, posteriormente se realizaron diluciones seriadas consecutivas y finalmente se determinó la concentración bacteriana de Staphylococcus aureus, Salmonella sp, Coliformes totales y fecales y esporas de clostridios sulfito reductor.

\section{Análisis sensorial}

El panel consistió de 30 jueces no entrenados que evaluaron las muestras codificadas. La evaluación sensorial (color, sabor, consistencia y aceptabilidad global) se basó en la escala hedónica de 10 puntos (1, me disgusta extremadamente; 10 me gusta extremadamente). Las muestras fueron presentadas en contenedores plásticos libres de olores, se utilizó agua para el lavado de la boca entre muestras. Se presentaron tres muestras a cada panelista.

\section{RESULTADOS Y DISCUSIÓN}

\section{Elaboración de las biopelículas}

Es posible obtener biopelículas activas con acción antimicrobiana utilizando proporciones (1:3) de agua destilada y hojas de toronjil; ya que se apreció un efecto antimicrobiano debido a la disminución del número 
de bacterias aerobias mesófilas en las muestras de camarón blanco, esta propiedad es atribuida a los compuestos presentes en el extracto acuoso de toronjil, dentro de los cuales se encuentran principalmente el Eugenol y Cariofileno, los cuales sobrepasan el $40 \%$ del total de los compuestos presentes y tienen efectos bactericidas, antifúngicos, antisépticos, antivirales, antioxidantes entre otras (García et al., 2010). La mayoría de los componentes que presentan mayor acción antimicrobiana son timol, el carvacrol, el linalool, el aldehído cinámico, la alicina y el eugenol (Montes et al., 2009), algunos encontrados en el toronjil (Acevedo et al., 2013). Resultados similares fueron reportados por Wu et al., (2014), quienes elaboraron una película a base de gelatina y quitosano incorporando aceite esencial de orégano, encontrando que la película sin la presencia de oregano no presentaba actividad antimicrobiana contra microorganismos de origen alimentario como: Escherichia coli, Staphylococcus aureus, Bacillus subtilis, Bacillus enteritidis y Shigabacillus. Sin embargo, cuando el aceite esencial de orégano fue adicionado a la película, esta presentó un marcado efecto antimicrobiano atribuido a la composición química del orégano, principalmente al Eugenol y Timol los cuales tienen propiedades bactericidas. Dichos compuestos por su acción lipofílica tienen la capacidad de pasar las membranas celulares, romper polisacáridos, ácidos grasos y lípidos, permeabilizando la membrana celular, lo cual inevitablemente conduce a la muerte celular; también se ha encontrado que a nivel citoplasmático pueden actuar sobre lípidos y proteínas coagulando tales moléculas (Bakkali et al., 2008). Las biopelículas obtenidas a base de alginato y almidón de maíz fueron visiblemente homogéneas, sin grumosidad y de fácil manejo, permitiendo una fácil aplicación en las muestras de camarón blanco, estas características se deben a las propiedades coloidales únicas del alginato y a su capacidad para formar geles fuertes, además también ha sido utilizado para reducir la microbiota bacteriana en alimentos (Rhim, 2004).

\section{Cinéticas de crecimiento de bacterias mesófilas aerobias}

Luego de realizadas las cinéticas de crecimiento de aerobios mesófilos fueron modeladas matemáticamente utilizando la ecuación de Baranyi y Roberts (1994) (ver figura 1). En las curvas de crecimiento microbiano es posible apreciar las distintas fases que determinan las curvas (fase de adaptación, logarítmica y estacionaria). Igualmente es importante destacar el efecto de las temperaturas de incubación $\left(17,27\right.$ y $\left.37^{\circ} \mathrm{C}\right)$ sobre la fase logarítmica principalmente.
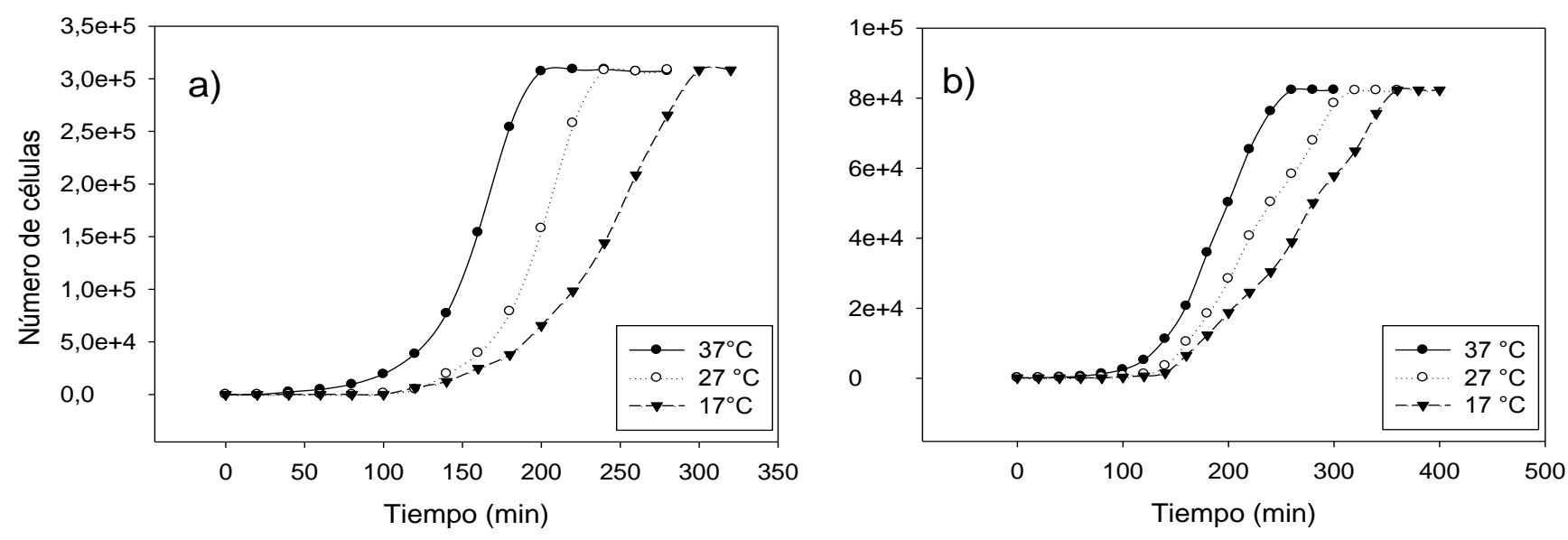

Fig.1: cinéticas de crecimiento a diferentes temperaturas a: sin biopelículas. b: con biopelículas

Como se muestra en la figura 1 en ambos tratamientos se apreciaron diferencias en la fase de adaptación, en cuanto a la etapa logarítmica se puede apreciar un incremento en la pendiente al aumentar la temperatura, este comportamiento es típico por tratarse de bacterias mesófilas. Igualmente, se observa que las bacterias presentes en las muestras de camarón blanco recubiertas con biopelículas activas entran en un mayor tiempo a la fase estacionaria, aunque generalmente este comportamiento es atribuido al agotamiento de los nutrientes presentes en el substrato, sin embargo no se debe descartar el mencionado efecto antimicrobiano que ejerce el extracto acuoso de toronjil.

De acuerdo a los resultados obtenidos en las cinéticas de crecimiento microbiano, se puede decir que fueron mayores a las reportadas por $\mathrm{Mu}$ et al., (2012) mostrando una diferencia equivalente a un $2 \%$ para el tratamiento control y un 4\% para el tratamiento con biopelículas; de igual forma Odilichukwu et al., (2014) mostraron un valor inicial de bacterias aerobias mesófilas de 1\% por debajo del tratamiento control y un $3 \%$ para el tratamiento con biopelículas. Probablemente, esto se debe a la diferencia de temperaturas manejadas en cada uno de los estudios descritos, los análisis a bajas temperaturas muestran una mayor reducción en el 
crecimiento de microorganismos aerobios mesófilos en comparación con análisis a temperatura ambiente $\left(37^{\circ} \mathrm{C}\right)$.

\section{Estimación de la vida útil microbiológica del camarón blanco}

La vida útil microbiológica de un producto alimenticio se puede estimar obteniendo los parámetros cinéticos de crecimiento microbiano a partir de la fase logarítmica, donde las células bacterianas se encuentran sanas y estables desde un punto de vista fisiológico. La velocidad de crecimiento específica $(\mu)$ para cada temperatura corresponde al valor de la pendiente de la recta de la fase logarítmica, mientras que el tiempo de duplicación ( $\mathrm{Td}$ ) es determinado por In2/ $\mu$. Posteriormente se grafican los valores de Td contra las temperaturas evaluadas, obteniendo la ecuación de la recta de la forma $Y=m x+b$; donde $x$ equivale al valor de la temperatura $\left(4^{\circ} \mathrm{C}\right)$ a la cual se predice la vida útil microbiológica. Finalmente el valor del $\mathrm{Td}$ final se reemplaza en la ecuación de Monod Hinshelwood. Los valores de Td se muestran en la tabla 1 en donde se puede observar un mayor Td para las bacterias en contacto con las biopelículas activas indicando que existe un retraso entre la aparición de la siguiente generación. Por ende, el extracto de Toronjil ejerce un efecto antimicrobiano, debido a su composición química, la cual ha sido previamente estudiada (Acevedo et al., 2013).

Considerando la variación de $\mu$ en función de la temperatura, es posible establecer una temperatura mínima por debajo de la cual no se evidencia crecimiento, a medida que la temperatura aumenta se produce un crecimiento lineal hasta que alcanzar la temperatura optima a la cual $\mu$ es máxima. Por encima de esta temperatura la velocidad de crecimiento decae bruscamente y se produce la muerte celular. Por otra parte, el aumento de $\mu$ con la temperatura se debe al incremento generalizado de la velocidad en las reacciones enzimáticas. En términos generales, la velocidad de las reacciones bioquímicas puede aumentar entre un 1.5 y 2.5 veces al elevarse $10^{\circ} \mathrm{C}$ la temperatura que tiene lugar (Ramírez, 2005). Como se mencionó previamente se utilizaron como microorganismos indicadores bacterias mesófilas aerobias, las cuales tienen una temperatura optima de crecimiento entre 30 y $45^{\circ} \mathrm{C}$ según lo reportado por Ramírez (2005); con base a esto es posible entender que a $37^{\circ} \mathrm{C}$ los microorganismos presentan su mayor tasa de crecimiento, lo que permite comprobar nuevamente el efecto bacteriostático ejercido por los componentes activos presentes en el extracto acuoso de Toronjil (Centeno y Carrera, 2013; Vizoso, 1997).

Tabla 1: vida útil microbiológica estimada para el camarón blanco a $4^{\circ} \mathrm{C}$

\begin{tabular}{|c|c|c|}
\hline parámetros & Sin biopelícula & Con biopelícula \\
\hline $\mathrm{Td}$ & $8.7 \mathrm{~h}$ & $18.3 \mathrm{~h}$ \\
\hline $\mathrm{Ts}$ & 11.6 días & 17.67 días \\
\hline $\mathrm{R}^{2}$ & 0.9985 & 0.9999 \\
\hline
\end{tabular}

Al obtener los datos de la cinética de crecimiento gracias al modelo de Baranyi y Robert y la aplicación de la ecuación de Monod-Hinshelwood, se determinó la vida útil microbiológica para cada tratamiento, resultados que se exponen en la tabla 1; a raíz de estos resultados es posible establecer un aumento del $33 \%$ en la vida útil microbiológica de los camarones cuando estos son recubiertos con biopelículas activas.

Los resultados de vida útil reportados en la presente investigación son mayores a los publicados por Odilichukwu et al., (2014), en los cuales reportan una vida útil de 8 días para camarones blanco del pacifico, esta diferencia puede ser ocasionada porque Odilichukwu et al., (2014) utilizaron camarones blancos frescos ( in la adición de sustancias antimicrobianas) almacenados en hielo y empacados en bolsas de polietileno y el método de estimación de vida útil estuvo basado en la determinación de acidez titulable, trimetilamina, diferencia de color, nitrógeno, valor de peróxido, anisidina y conteo de microorganismos aerobios mesófilos.

Con respecto al crecimiento de bacterias mesófilas aerobias, es indiscutible que el deterioro por este tipo de bacterias incrementa con la muerte del camarón. Esto se debe al colapso del sistema inmunológico cuando el camarón muere y las bacterias proliferan rápidamente. En el presente estudio, todas las muestras de camarón fueron decapitadas antes de los análisis y esto pudo influenciar el grado de proliferación de las bacterias. Ha sido reportado que la decapitación puede potencialmente reducir el número de bacterias en cierto grado. También el efecto de las bacterias aerobias está mayoritariamente asociado con los camarones frescos e incluyen géneros bacterianos como Pseudomonas, Achromobacter, Flavobacterioum así como Micrococcus (Lu, 2009).

Tal y como lo mencionó Dalgaard y Jorgensen, (2000), los modelos matemáticos usados para predecir la vida útil de productos marinos pueden generar diferencias dependiendo de la composición del producto y a factores 
externos. El modelo común (basado en el modelo de Ratkowsky) para la predicción de la vida útil de mariscos; vida útil $(\mathrm{T})=$ vida útil $\left(\mathrm{T}_{0}\right) /(1+0,1 \times \mathrm{T})^{2}$ (Dalgaard and Huss, 1997) predice una vida útil de 16,5 h para una vida útil de $11 \mathrm{~d} \mathrm{a} 0^{\circ} \mathrm{C}$. Esta predicción es poco realista. La ecuación falla en la exactitud de la predicción cuando ocurre un incremento de la temperatura debido al cambio de la microbiota dominante de baja temperatura a elevada temperatura (Dalgaard and Huss, 1997; Dalgaard and Jorgensen, 2000).

\section{Análisis microbiológicos}

Los resultados de los análisis microbiológicos llevados a cabo sobre las biopelículas revelaron que las biopelículas no fueron fuente de contaminación bacteriana como consecuencia del calentamiento $\left(90^{\circ} \mathrm{C} / 10\right.$ min) al que son sometidas durante el proceso de elaboración. Igualmente, se puede apreciar en la tabla 2 que el camarón blanco utilizado presenta buena calidad microbiológica y por ende recomendable su consumo.

Tabla 2: calidad microbiológica de las biopelículas activas

\begin{tabular}{|l|c|c|}
\hline \multicolumn{1}{|c|}{ Tipo de microorganismo evaluado } & Biopelículas activas & Camarón blanco \\
\hline S.aureus Coagulasa $(+)$ & Ausencia & Ausencia \\
\hline Salmonella $\mathrm{sp}$ & Ausencia en $25 \mathrm{~g}$ de muestra & Ausencia en $25 \mathrm{~g}$ de muestra \\
\hline Coliformes totales & $<3 \mathrm{bact} / \mathrm{gr}$ & $<3 \mathrm{bact} / \mathrm{gr}$ \\
\hline Coliformes fecales & $<3 \mathrm{bact} / \mathrm{gr}$ & $<3 \mathrm{bact} / \mathrm{gr}$ \\
\hline Esporas de clostridios reductores de sulfito & $<10 \mathrm{UFC} / \mathrm{g}$ & $<10 \mathrm{UFC} / \mathrm{g}$ \\
\hline
\end{tabular}

\section{Evaluación sensorial de los Camarones}

Las pruebas sensoriales fueron llevadas a cabo a los 8 días de almacenamiento de las muestras de camarón evaluadas. Los promedios de los puntajes obtenidos por todos los panelistas se presentan en la figura 2 . Los resultados muestran que no se encontraron variaciones en los atributos evaluados entre las muestras de camarón blanco recubierto, sin recubrir y una muestra control almacenada en bolsas de polietileno de baja densidad.

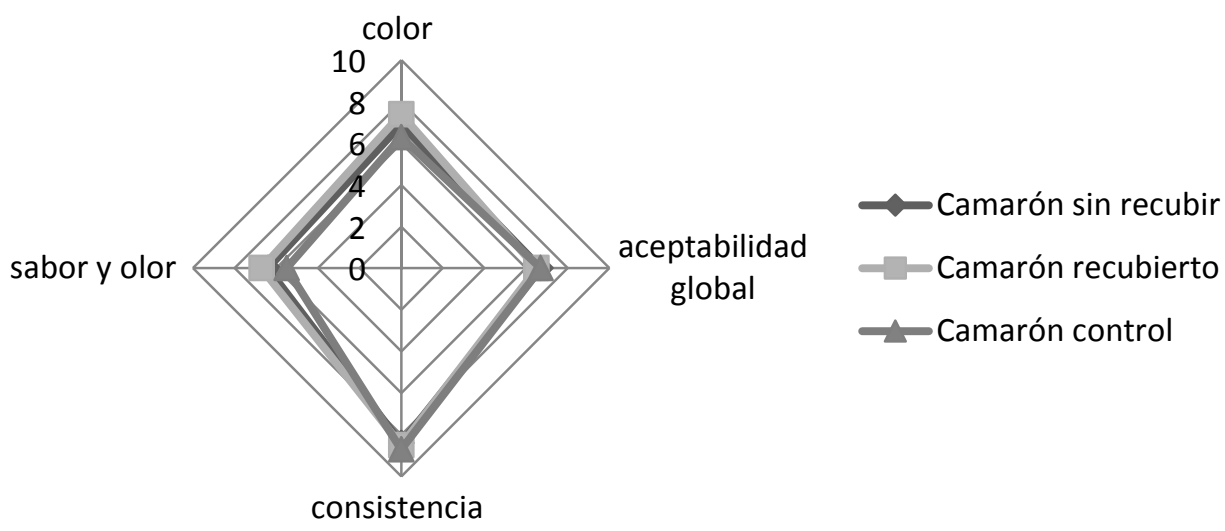

Fig. 2: Análisis sensorial de las muestras de Camarón evaluadas

Se esperaba que la incorporación de extracto acuoso de toronjil en las biopelículas afectara negativamente los atributos sensoriales, especialmente el sabor y olor. Sin embargo, los panelistas no lograron establecer diferencias entre las muestras, este comportamiento posiblemente se debe al carácter volátil de la mayoría de compuestos olfatorios presentes en el toronjil, los cuales pueden no encontrarse al finalizar la vida útil del camarón. Dabadé et al., (2015) establecieron como tiempo de rechazo del camarón (Penaeus notialis) cuando al menos el $50 \%$ de los panelistas detectaba la producción de olores fuertes por parte del marisco, encontrando la liberación de estos olores a las $10 \mathrm{~h}$ a $28^{\circ} \mathrm{C}$, de $4 \mathrm{~d}$ a $7^{\circ} \mathrm{C}$ y de $12 \mathrm{~d}$ a $0^{\circ} \mathrm{C}$. Claramente estos resultados no concuerdan con los reportados en esta investigación. Dicha discrepancia puede ser ocasionada porque Dabadé et al., (2015) no aplicaron ningún tipo de tratamiento al camarón con el fin de incrementar la vida útil del mismo. Lo cual pone en evidencia nuevamente, el efecto de las biopelículas activas a base de polímeros y extracto acuoso de toronjil en la prolongación de la vida útil de productos alimenticios sin afectar las propiedades sensoriales de los alimentos. 


\section{CONCLUSIONES}

Es posible obtener biopelículas activas a base de almidón de maíz y alginato utilizando como componente natural antimicrobiano el extracto acuoso de toronjil. La presencia de las biopelículas activas logra aumentar el tiempo de duplicación de las bacterias mesófilas aerobias que crecen en el camarón blanco, lo cual conlleva a incrementar el tiempo de vida útil microbiológica un 33\% en comparación con los camarones que no fueron recubiertos. Por tal motivo, la utilización de biopelículas a base de materiales poliméricos se vislumbra como una técnica para incrementar la vida útil de productos alimenticios y es una alternativa al uso tradicional de materiales termoplásticos para el envasado de alimentos.

\section{REFERENCIAS}

Acevedo, D. y otros dos autores, Composición Química del Aceite Esencial de las Hojas de Toronjil (Melissa officinalis L.), Inf Tecn, 24 (4), 49-54 (2013)

Bakkali, S. y otros tres autores, Biological effects of essential oils: a review. Food Chem Tox, 46, 446-475 (2008)

Baranyi, J. y T. Roberts, A dynamic approach to predicting bacterial growth in food. Int. J. Food Microbiol. 23, 277-294 (1994)

Bono, G. y C. Badalucco, C. Combining ozone and modified atmosphere packaging (MAP) to maximize shelflife and quality of striped red mullet (Mullus surmuletus). LWT Food Sci Technol, 47(2), $500-504$ (2012).

Cadun, A. y otros dos autores, A study of marination of deepwater pink shrimp (Parapenaeus longirostris, Lucas, 1846) and its shelf life, Food Chem, 90, 53-59 (2005)

Castillo, F y otros cuatro autores, Freshness loss in sierra fish (Scomberomorus sierra) muscle stored in ice as affected by postcapture handling practices, J. Food Biochem, 31(1), 56-67 (2007)

Centeno S, y Y. Carrera, Actividad antifúngica y antiaflatoxigénica de extractos de Melissa officinalis (lamiaceae) sobre aspergillus flavus, Saber, 25(2), 13-25 (2013)

Chana-Thaworn, J. y otros dos autores, Properties and antimicrobial activity of edible films incur-porated with kiam wood (Cotyleobium lanceotatum) extract. Food Sci and Technol, 44(1), 284-292. (2011)

Chen, J. y otros dos autores, Preparation of a novel botanic biopreservative and its efficacy in keeping quality of peeled Penaeus vannamei, Food Sci. Technol. Int. 19, 251-260 (2013)

Dabadé, S. y otros cinco autores, Spoilage evaluation, shelf-life prediction, and potential spoilage organisms of tropical brackish water shrimp (Penaeus notialis) at different storage temperatures, Food Microbiol, 48 8-16 (2015)

Dalgaard, P. y L. Jorgensen, Cooked and brined shrimps packed in a modified atmosphere have a shelf-life of $>7$ months at $0^{\circ} \mathrm{C}$, but spoil in 4-6 days at $25^{\circ} \mathrm{C}$, Int. J. Food Sci. Technol. 35, 431-442 (2000)

Dalgaard, P. y H. Huss, Mathematical modeling used for evaluation and prediction of microbial fish spoilage. In: Shahidi, F., Jones, Y., Kitts, D.D. (Eds.), Seafood Safety, Processing and Biotechnology, Technomic pub. Co. Inc, Lancaster, PA, pp. 73-89 (1997)

Esquivel, A. y P. Vargas, Uso de aceites esenciales extraídos por medio de fluidos supercríticos para la elaboración de alimentos funcionales, Tecnol en Marcha, 20(4), 41-50 (2007)

Embuscado, M. y Huber, K. Edible films and Coatings for food Applications. Springer, London New York, (2009)

García, C. y otros dos autores, Elaboración de Biopeliculas a partir del nejayote de maíz (Zea mays) y almidón de sorgo (Sorghum bicolor Moench) aplicadas en fresa (Fragaria vesca), Universidad Politécnica de Guanajuato, Mexico, (2010)

González, R. y otros dos autores, Efecto de la microencapsulación sobre las propiedades reológicas y fisicoquímicas del yogurt blando, Inf. Tecnol, 25 (6), 45-56 (2014)

Herrera, F. y R. García, Evaluación in vitro del efecto bactericida de extracto acuso de laurel, clavo, canela, y tomillo sobre cinco cepas bacterianas patógenas de origen alimentario, Rev Bistua, 4 (2), 13-19 (2006) 
Jiménez, A, y otros cuatro autores, Influence of nanoliposomes incorporation on properties of film forming dispersions and films based on corn starch and sodium caseinate, Food Hydrocol, 35, 159-169 (2014)

Kuorwel, K. y otros cinco autores, Antimicrobial Activity of Biodegradable Polysaccharide and Protein-Based Films Containing Active Agents, J Food Sci, 76, 90-110 (2011)

Lee, K. y D. Mooney, Alginate: Properties and biomedical applications. Progr Pol Sci, 37(1) 106-126 (2012)

Liu, Z, Edible films and coatings from starches. En: Innovations in food packaging, Jung H. Han (ed). Elsevier, Amsterdam, The Netherlands. (2005)

Lu, S. Effects of bactericides and modified atmosphere packaging on shelf-life of Chinese shrimp (Fenneropenaeus chinensis), Food Sci Technol, 42(1), 286-291 (2009)

Montes, R. Diversidad de compuestos químicos producidos por las plantas contra hongos fitopatógenos: revisión, Rev Mex Micol, 2974 - 82 (2009)

$\mathrm{Mu}, \mathrm{H}$. y otros cuatro autores, Effect of cinnamaldehydeon melanosis and spoilage of Pacific white shrimp (Litopenaeus vannamei) during storage, J. Sci Food Agri, 92, 2177-2182 (2012)

Odilichukwu, C. y otros dos autores, Quality and shelf life assessment of Pacific white shrimp (Litopenaeus vannamei) freshly harvested and stored on ice, Food Sci and Technol, 55, 110-116 (2014)

Ocano, V. y otros autores, Postmortem changes in cazon fish muscle stored on ice, Food Chem, 116(4), 933938 (2009)

Ramírez L, Crecimiento y muerte de microorganismos, Genetic and Microbiologica Group, Universidad de Navarra (España) (2005)

Rhim, J. Physical and mechanical properties of water resistant sodium alginate films. LWT-Food Sci Technol, 37(3), 323-330 (2004)

Rodríguez, D. y R. Schobitz, Película antimicrobiana a base de proteína de suero lácteo, incorporada con bacterias lácticas como controlador de Listeria monocytogenes, aplicada sobre salmón ahumado, Rev. Biotecnol, Sec Agrop Agroind, 7(2), 49 - 54 (2009)

Shen, X.L., Wu, J., Chen, Y. y G. Zhao, Antimicrobial and physical properties of sweet potato starch films incorporated with potassium sorbate or chitosan, Food Hydrocol, 24(4), 285-290. (2010)

Vizoso, P. y otros cuatro autores, Estudio genotóxico in vitro e in vivo en tinturas de Melissa officinalis L. (toronjil) y Mentha piperita L. (toronjil de menta), Rev Cubana Plant Med, 2(1) (1997)

Wu, J. y otros siete autores, Properties and antimicrobial activity of silver carp (Hypophthalmichthys molitrix) skin gelatin-chitosan films incorporated with oregano essential oil for fish preservation, 2(1), 7-16 (2014)

Zeng, Q. y otros dos autores, Quality changes of shrimp (Pandalus borealis) stored under different cooling conditions, J. Food Sci. 70, S459-S466 (2005) 